\title{
A 3D Shear Material Damping Model for Man-Made Vibrations of the Ground
}

\author{
Darius Macijauskas ${ }^{1}$, Stefan Van Baars ${ }^{2}$ \\ ${ }^{I}$ ArcelorMittal Commercial RPS, Esch-sur-Alzette, Luxembourg \\ ${ }^{2}$ Faculty of Science, Technology and Communication, University of Luxembourg, Luxembourg, Luxembourg \\ E-mails: 'darius@macijauskas.net (corresponding author); ${ }^{2}$ stefan.vanbaars@uni.lu
}

\begin{abstract}
In this article, a 3 dimensional shear damping constitutive soil model is presented. This model has been implemented as a user defined soil model for the Finite Element Method software of Plaxis 2D. Verification tests demonstrate a good agreement with the theoretical model. A pulse load on a circular area on a soil surface has been numerically modelled with this shear damping soil model and compared to the results obtained by using the Rayleigh damping. The shear damping model demonstrates a different physical behaviour of the soil, in comparison to the Rayleigh damping.
\end{abstract}

Keywords: Soil vibration, man-made vibrations, soil damping, material damping, wave propagation.

Conference topic: Design experiences and theoretical solutions.

\section{Introduction}

One important aspect of soil dynamics is the material damping of vibrations. Some authors like Richart et al. (1970) state that the material damping is not as important as the radiation damping, but others, like Wolf (1985), Sienkiewicz (1993) and Ambrosini (2006) highlight the importance of it.

For problems of dynamic soil-structure interaction, the soil behaviour is often modelled in terms of masses, springs and dashpots. If a more accurate description of the soil behaviour is needed, the Finite Element Modelling (FEM) technique can be used. In FEM, the material damping is usually modelled by applying a viscous damping, such as the Rayleigh damping. Viscous damping is unfortunately frequency dependent, which is not the case for the physical behaviour of soil itself. Abbiss (1986) claimed soils to be viscous; others, like Iwasaki et al. (1978), Bolton, Wilson (1990) and Van Baars (2011), stated that the material damping is not viscous, but hysteretic. The resulting problem of viscous damping can be illustrated by considering a superposition of waves with multiple frequencies, for which there can be no equivalent viscosity for all waves at the same time (Van Baars 2011). Bolton, Wilson (1990) proved with tests on dry and saturated sand that up to $100 \mathrm{~Hz}$, the material damping is hysteretic and strain dependent for higher strains, but independent of the frequency. The fact that material damping is independent of the strains in the small strain zone and independent of the frequency in the low frequency range, is also supported by Karl et al. (2006).

For larger strains, the soil damping depends on the shear strain amplitude (Okur, Ansal 2007). The bigger the shear strain amplitude, the bigger the amount of energy which is damped, see Figure 1. This behaviour has been implemented in the Hardening Soil small- strain stiffness model (HSSmall) by Benz (2007). The model is available in the FEM software Plaxis. Very important is that this constitutive soil model has a frequency independent damping. However, Brinkgreve et al. (2007) concluded, that HSsmall model does not capture material damping at small strain levels. It means that for the moment, there is no frequency independent constitutive material damping model available for the small strain range. This model was originally made for static analysis, for which the definition of small strains is different. In this article, the small strain range definition is used for strains which are smaller than $10^{-4}$.

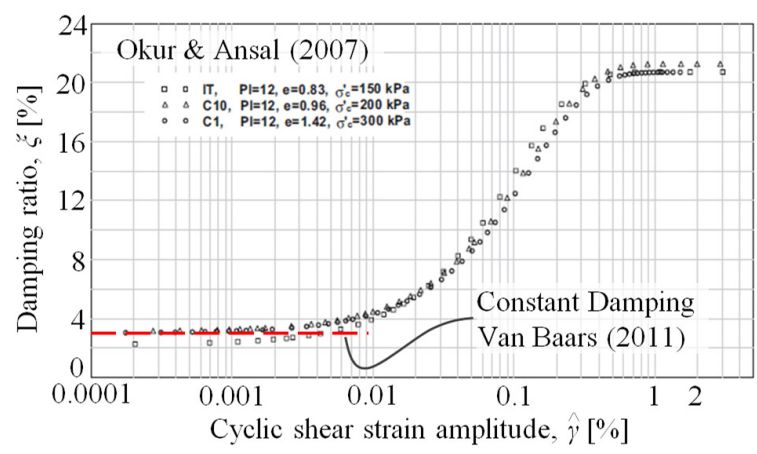

Fig. 1. Damping ratio dependence on cyclic shear strain amplitude of natural fine grained soils

Van Baars (2011) suggested to distinguish between large vibrations, such as earthquakes, and small vibrations, such as man-made vibrations. Man-made vibrations are small vibrations, caused by different human activities, like machine vibrations, pile driving, explosions, trains, etc. Nevertheless, they can disturb people or vibration sensitive industrial processes or cause damage to historical, sensitive buildings. 
Measurement data of Okur and Ansal (2007) shows that for shear strains smaller than $10^{-4}$ the damping ratio is nearly constant. Van Baars (2011) proposed an exponential shear damping model and presented it in 1D form. The general idea of this model is to have a constant damping ratio independently from the shear strain amplitude. This is exactly what is needed for man-made vibrations. The model's behaviour is based on the hypothesis that the material damping is caused by friction between particles and this friction occurs mostly due to shear deformation rather than volume. The energy in a numerical model should not be dissipated by a damping matrix [C], but by a cyclic nonlinear stress strain behaviour. Such a constitutive model can be classified as a cyclic nonlinear model, according to the classification suggested by Kramer (1996).

The aim of this article is to demonstrate that it is possible to damp small strains physically in a correct manner by using a conventional geotechnical tool, like Plaxis. In order to achieve this, first of all, the 1D shear damping model, proposed by Van Baars (2011), will be explained in detail and later be extended for a 3D case. Afterwards, a procedure of implementing the 3D shear damping model into Plaxis software, as a user defined soil model (UDSM), is briefly described. In order to check whether the model was implemented correctly and whether the iterative procedures of Plaxis can converge with this unusual constitutive model, two types of verification tests will be presented. Finally, a numerical problem of a pulse load on a circular area on the soil surface will be described and solved with this shear damping model and compared to the results obtained by using the Rayleigh damping.

\section{Shear damping}

\section{One dimensional}

The original shear damping model was described for a one dimensional case in the publication of Van Baars (2011).

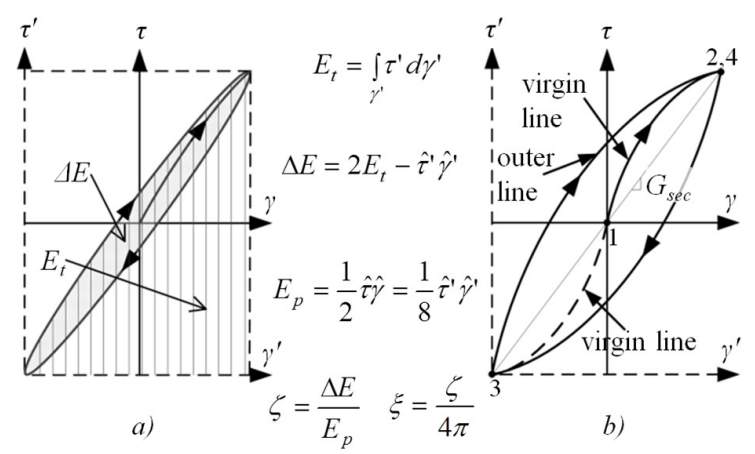

Fig. 2. One dimensional shear damping law

The used shear damping law, in a $\tau^{\prime}-\gamma^{\prime}$ coordinate system, was defined as follows:

$$
\tau^{\prime}=G_{\bmod }\left(\gamma^{\prime}\right)^{X}
$$

where: $\tau^{\prime}$ - the shear stress since the start, or the last change of load direction; $G_{\text {mod }}$ - the modified shear modulus; $\gamma^{\prime}-$ is the shear strain since the start, or the last change of load direction and $X$ is the dimensionless damping parameter, which is positive and smaller than or equal to 1.

The damping parameter $X$ is related to the damping ratio $\xi$ or to the energy ratio $\zeta$, which can be measured directly by using various types of laboratory tests (cyclic simple shear test, cyclic torsional simple shear test, cyclic triaxial test or resonant column test):

$$
X=\frac{8-\zeta}{8+\zeta}=\frac{2-\pi \xi}{2+\pi \xi} .
$$

For the damping ratio $\xi=0$, the dimensionless damping parameter $X=1$, so the shear stress can be calculated as $\tau^{\prime}=G_{\text {mod }} \gamma^{\prime}$. In this case $G_{\text {mod }}=G_{\text {sec }}$, where $G_{\text {sec }}$ is a secant modulus for the full cycle (see $b$ ) in Figure 2). However if $\xi>0$, then the modified shear modulus $G_{\text {mod }}$ depends on the shear strain amplitude (see $b$ ) in Figure 2), so $G_{\text {mod }} \neq G_{\text {sec }}$. The modified shear modulus $G_{\text {mod }}$ can be related to $G_{\mathrm{sec}}$ as follows:

$$
\frac{G_{\mathrm{mod}}}{G_{\mathrm{sec}}}=\frac{2 \hat{\gamma}}{(2 \hat{\gamma})^{X}} .
$$

The relationship between $G_{\text {mod }}$ and $G_{\text {sec }}$ for different damping ratios and different shear strain ranges can be seen in Figure 3.

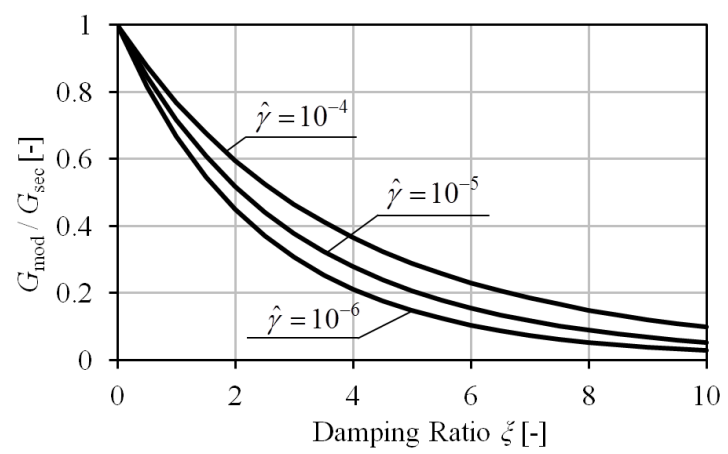

Fig. 3. The relationship between the modified and secant shear moduli

In order to form a constitutive model, the stresses and strains have to be defined in the $\tau-\gamma$ coordinate system, rather than in the $\tau^{\prime}-\gamma^{\prime}$. From Figure 2, it can be seen, that for multiple constant cycles, $\hat{\gamma}^{\prime}=2 \hat{\gamma}$ and $\hat{\tau}^{\prime}=2 \hat{\tau}$.Therefore, the virgin damping line in the $\tau-\gamma$ coordinate system, can be expressed as follows:

$$
\tau_{v i r}=\frac{G_{\bmod }(2 \gamma)^{X}}{2} .
$$

In order to calculate the outer lines (see b) in Fig. 2) in the $\tau-\gamma$ coordinate system, the direction of the shear-strain path must be known as well as the peaks 
of the last direction change. Then the shear stresses of the outer lines are calculated as follows:

$$
\begin{gathered}
\tau_{\text {out }, \text { up }}=\tau_{\text {min }}+G_{\text {mod }}\left|\gamma-\gamma_{\text {min }}\right|^{X} ; \\
\tau_{\text {out }, \text { down }}=\tau_{\text {max }}-G_{\text {mod }}\left|\gamma-\gamma_{\max }\right|^{X},
\end{gathered}
$$

where: $\tau_{\text {out,up }}$ and $\tau_{\text {out,down }}$ are the shear stresses of the rising and descending outer line respectively; $\tau_{\min }$ and $\tau_{\max }$ are the stored peak stresses: minimum and maxishear strains: minimum and maximum respectively.

The outer lines draw an ellipse shaped figure in $\tau-\gamma$ coordinates, which represents the dissipated energy mum respectively; $\gamma_{\min }$ and $\gamma_{\max }$ are the stored peak

per volume, per cycle. By using this law, the dissipated shear strain energy ratio $\zeta$ and the damping ratio $\xi$ are independent from the shear strain amplitude.

\section{Three dimensional}

Before being able to extend this 1D shear damping model to a 3D model, it should be rewritten. In 1D there is only a pure shear stress and strain. In general, shear strains change only the shape, but not the volume. For $3 \mathrm{D}$, however, the linear stress-strain relationship, or Hooke's law, is expressed by the modulus of elasticity $E$ and the Poisson's ratio $v$, as follows:

$$
\left\{\begin{array}{l}
\sigma_{x x} \\
\sigma_{y y} \\
\sigma_{z z} \\
\sigma_{x y} \\
\sigma_{y z} \\
\sigma_{z x}
\end{array}\right\}=\frac{E}{(1+v)(1-2 v)}\left[\begin{array}{cccccc}
1-v & v & v & 0 & 0 & 0 \\
v & 1-v & v & 0 & 0 & 0 \\
v & v & 1-v & 0 & 0 & 0 \\
0 & 0 & 0 & \frac{(1-2 v)}{2} & 0 & 0 \\
0 & 0 & 0 & 0 & \frac{(1-2 v)}{2} & 0 \\
0 & 0 & 0 & 0 & 0 & \frac{(1-2 v)}{2}
\end{array}\right]\left\{\begin{array}{l}
\varepsilon_{x x} \\
\varepsilon_{y y} \\
\varepsilon_{z z} \\
\gamma_{x y} \\
\gamma_{y z} \\
\gamma_{z x}
\end{array}\right\},
$$

where: $\sigma_{i i}$ and $\varepsilon_{i i}$ are normal stresses and strains respectively, $\sigma_{i j}$ and $\gamma_{i j}$ are shear stresses and strains respectively, in which $i, j=x, y$ or $z$ and $i \neq j$.

The sign convention for the stresses and strains are used according to Plaxis' Material Models Manual (Brinkgreve et al. 2015), which is presented in Figure 4.
In a $3 \mathrm{D}$ case, there are three pure shear strains and three corresponding pure shear stresses. However, they are not the only ones, responsible for the change of the shape. This can be illustrated by decomposing Hook's law into two parts: the deviatoric part, controlled by the shear modulus $G$, and the volumetric part, controlled by the bulk modulus $K$ :

$$
\left\{\begin{array}{l}
\sigma_{x x} \\
\sigma_{y y} \\
\sigma_{z z} \\
\sigma_{x y} \\
\sigma_{y z} \\
\sigma_{z x}
\end{array}\right\}=G\left[\begin{array}{cccccc}
\frac{4}{3} & -\frac{2}{3} & -\frac{2}{3} & 0 & 0 & 0 \\
-\frac{2}{3} & \frac{4}{3} & -\frac{2}{3} & 0 & 0 & 0 \\
-\frac{2}{3} & -\frac{2}{3} & \frac{4}{3} & 0 & 0 & 0 \\
0 & 0 & 0 & 1 & 0 & 0 \\
0 & 0 & 0 & 0 & 1 & 0 \\
0 & 0 & 0 & 0 & 0 & 1
\end{array}\right]\left\{\begin{array}{l}
\varepsilon_{x x} \\
\varepsilon_{y y} \\
\varepsilon_{z z} \\
\gamma_{x y} \\
\gamma_{y z} \\
\gamma_{z x}
\end{array}\right\}+K\left[\begin{array}{cccccc}
1 & 1 & 1 & 0 & 0 & 0 \\
1 & 1 & 1 & 0 & 0 & 0 \\
1 & 1 & 1 & 0 & 0 & 0 \\
0 & 0 & 0 & 0 & 0 & 0 \\
0 & 0 & 0 & 0 & 0 & 0 \\
0 & 0 & 0 & 0 & 0 & 0
\end{array}\right]\left\{\begin{array}{l}
\varepsilon_{x x} \\
\varepsilon_{y y} \\
\varepsilon_{z z} \\
\gamma_{x y} \\
\gamma_{y z} \\
\gamma_{z x}
\end{array}\right\} .
$$

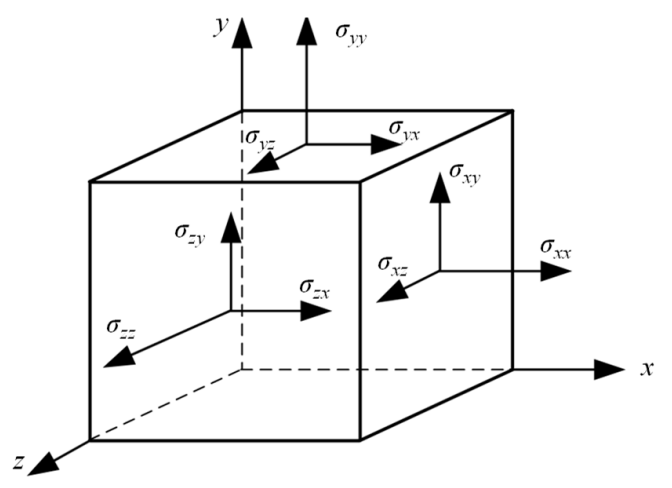

Fig. 4. Sign convention for stresses and strains
From Equation (8), it can be seen that for the change of the volume, which is controlled by $K$, the three normal stresses or strains are responsible. However, for the change of the shape, which is controlled by $G$, all six strains or stresses are responsible.

The deviatoric strains, which are responsible for the change of the shape, may be expressed as follows:

$$
\begin{aligned}
& e_{i i}=\varepsilon_{i i}-\frac{1}{3} \varepsilon_{v o l}, \\
& e_{i j}=\gamma_{i j},
\end{aligned}
$$

where: $\varepsilon_{v o l}=\varepsilon_{x x}+\varepsilon_{y y}+\varepsilon_{z z}$ is the total volumetric strain and $i, j=x, y$ or $z$ and $i \neq j$. 
The corresponding deviatoric stresses are calculated by:

$$
\begin{aligned}
& \tau_{i i}=\sigma_{i i}-\sigma_{0}, \\
& \tau_{i j}=\sigma_{i j},
\end{aligned} .
$$

where: $\sigma_{0}=\frac{1}{3}\left(\sigma_{x x}+\sigma_{y y}+\sigma_{z z}\right)$ is the isotropic stress and $i, j=x, y$ or $z$ and $i \neq j$.

The shear damping law for a $3 \mathrm{D}$ case can be applied by using Eq. (1) in $\tau^{\prime}-e^{\prime}$ coordinates:

$$
\begin{aligned}
\tau^{\prime}{ }_{i i} & =G_{\bmod }\left(e^{\prime}{ }_{i i}\right)^{X}, \\
\tau^{\prime}{ }_{i j} & =G_{\bmod }\left(e^{\prime}{ }_{i j}\right)^{X} .
\end{aligned}
$$

With this, the Cartesian stresses can be calculated:

$$
\begin{aligned}
\sigma_{i i} & =2 \tau_{i i}+K \varepsilon_{v o l}, \\
\sigma_{i j} & =\tau_{i j} .
\end{aligned}
$$

\section{Damped energy in a three dimensional case}

The damped energy in a three dimensional case cannot be so easily demonstrated graphically as it was done for the one dimensional case and presented in Figure 2.

The full potential strain energy per volume can be mathematically expressed as follows:

$$
E_{0}=0.5\left(\begin{array}{l}
\sigma_{x x} \varepsilon_{x x}+\sigma_{y y} \varepsilon_{y y}+\sigma_{z z} \varepsilon_{z z} \\
+\sigma_{x y} \gamma_{x y}+\sigma_{y z} \gamma_{y z}+\sigma_{z x} \gamma_{z x}
\end{array}\right) \text {. }
$$

The full potential strain energy per volume is the sum of the potential strain energy which changed the volume $E_{0 \mathrm{~V}}$ and the potential strain energy which changed the shape $E_{0 S}$. Therefore, Eq. (13) may be rewritten as follows:

$$
E_{0}=E_{0 V}+E_{0 S} .
$$

The potential strain energy per volume, responsible for the change of the volume, is expressed as:

$$
E_{0 V}=0.5 \varepsilon_{v o l} \sigma_{0} .
$$

The potential strain energy per volume, responsible for the change of the shape, can be written as:

$$
E_{0 S}=0.5\left(\begin{array}{l}
\tau_{x x} e_{x x}+\tau_{y y} e_{y y}+\tau_{z z} e_{z z} \\
+\tau_{x y} e_{x y}+\tau_{y z} e_{y z}+\tau_{z x} e_{z x}
\end{array}\right)
$$

The potential strain energy, responsible for the change of the volume, may be called the volumetric potential strain energy. In the same way, the potential strain energy, responsible for the change of the shape, may be called the deviatoric potential strain energy.

Due to the shear damping, a part of the deviatoric potential strain energy is dissipated. The ratio of the dissipated deviatoric strain energy to the deviatoric potential strain energy can be called the dissipated devia- toric strain energy ratio $\zeta=\Delta E_{S} / E_{0 S}$. With this, the shear damping ratio $\xi$ can be expressed as:

$$
\xi=\frac{\zeta}{4 \pi}=\frac{1}{4 \pi} \frac{\Delta E_{S}}{E_{0 S}} .
$$

Here it should be noted, that in the shear damping model, the shear damping ratio is not related to the full potential strain energy but only to its deviatoric part.

\section{User defined soil material model}

This shear damping constitutive model is implemented into the FEM software of Plaxis, as a user defined soil model (UDSM).

During the first iteration of a loading step, Plaxis provides strains which are calculated assuming linear elasticity. Then, using these provided strains, the stresses are determined according to the user provided constitutive model. The external forces are known and the internal forces are calculated from the constitutive stresses. In a next step, the unbalance between these internal and external forces is checked. If the error is bigger than tolerated, a new (Newton-Raphson) iteration process is started. The (Newton-Raphson) iterations are repeated for a loading step, until the tolerance of the unbalance error is reached (usually 0.01).

The UDSM consists of the following steps: 1) calculation of the deviatoric strains according to Eq. (9); 2) checking the stress-strain path direction; 3) checking the stress-strain amplitudes; 4) calculation of the deviatoric stresses according to Eq. (11); 5) calculation of the Cartesian stresses according to Eq. (12).

\section{Verification tests}

Two verification tests are performed: 1) a cyclic simple shear test, and 2) a cyclic triaxial test. For both verification tests, displacement controlled loading is used. The objective of the verification tests is to check whether the UDSM is well implemented in Plaxis and gives the same results as the analytical formulation of the constitutive model.

The verification tests also checks if the iterative procedure of Plaxis can converge with this unusual constitutive model, where the bulk and deviatoric deformation parts are separated and the latter is forced to have plastic deformations in order to damp the energy.

The cyclic simple shear tests are modelled in plain strain conditions, and the cyclic triaxial tests in axisymmetric conditions.

The following soil properties for the verification tests are chosen: the modified shear modulus $G_{\text {mod }}=100 \mathrm{kN} / \mathrm{m}^{2}$ and the bulk modulus $K=$ $166.67 \mathrm{kN} / \mathrm{m}^{2}$. Two different damping ratios are used: $\xi=5 \%$ and $10 \%$. Such high damping ratios are only selected in order to have wider loops in the plots.

The comparison between the results of the verification tests obtained by the UDSM, and by the analyti- 
cal laws, can be seen in Figure 5 and 6, for cyclic simple shear tests and cyclic triaxial tests respectively.

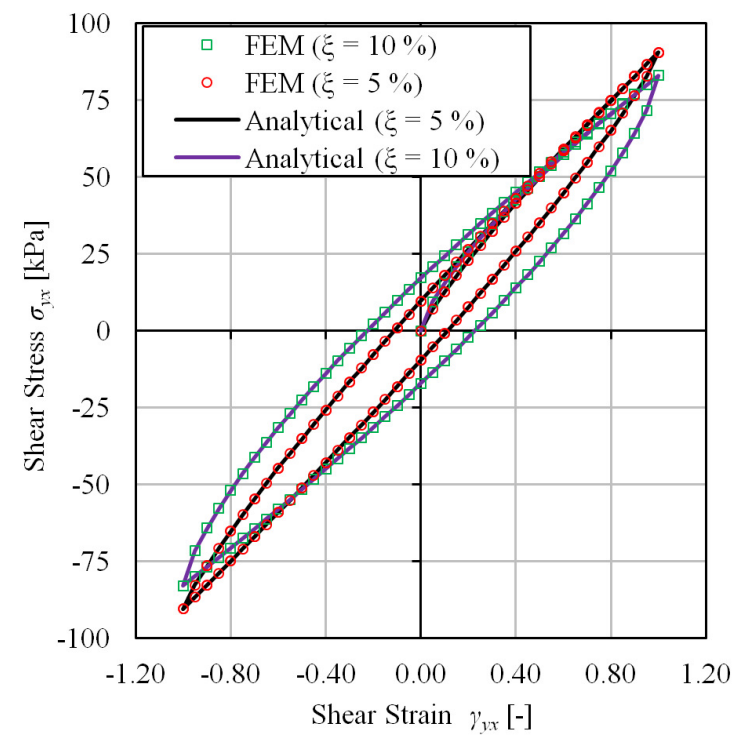

Fig. 5. Results of the verification tests with UDSM in Plaxis $2 \mathrm{D}$ for cyclic simple shear test

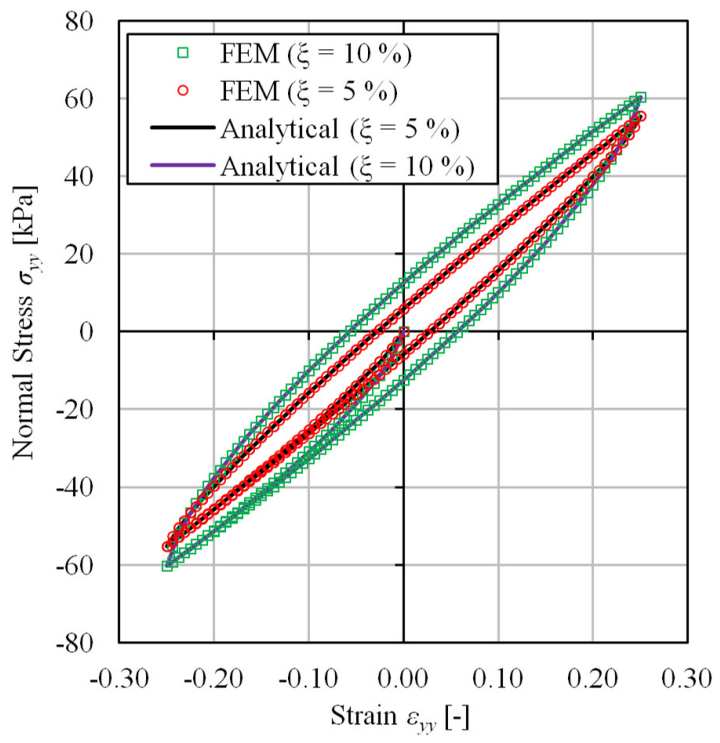

Fig. 6. Results of the verification tests with UDSM in Plaxis 2D for cyclic triaxial test

The results are for the different shear damping ratios in good agreement with the analytical shear damping model laws: Eqs (4), (5) and (6).

\section{Rayleigh damping versus shear damping}

\section{FE model parameters}

The Rayleigh damping (available in Plaxis), is unfortunately frequency dependent and damps deformations of all wave types, instead of only the deviatoric part. In order to study the effect of this, the Rayleigh damping will be compared to the shear damping model, which does not have these problems. For this, a pulse load on a circular area on the soil surface is modelled numerically. The pulse load of $2 \mathrm{kN}$ is modelled as a maximum vertical stress of $63.66 \mathrm{kN} / \mathrm{m}^{2}$ over an area at the soil surface with a diameter of $0.2 \mathrm{~m}$. The vertical stress is introduced in a time period from 0 to $0.005 \mathrm{~s}$, with its peak at $0.0025 \mathrm{~s}$. The geometry is presented in Figure 7.

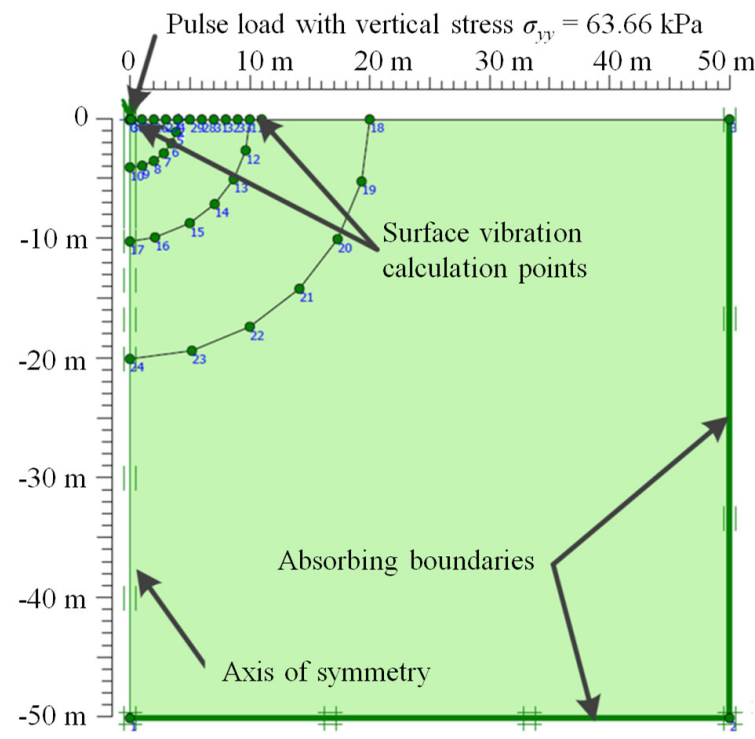

Fig. 7. FE model geometry

An axisymmetric mesh with a length and depth of $50 \mathrm{~m}$ is used. The dynamic problem is calculated first without damping, second with Rayleigh damping and third with shear damping. A damping ratio of $\xi=3 \%$ will be used for both the Rayleigh and shear damping.

The compressional wave velocity of the soil medium $v_{p}=200 \mathrm{~m} / \mathrm{s}$, the shear wave velocity $v_{s}=60 \mathrm{~m} / \mathrm{s}$ and the density $\rho=1.5 \mathrm{t} / \mathrm{m}^{3}$. From this follows the small strain shear modulus $G_{0}=\rho \cdot v_{s}^{2}=5400 \mathrm{kN} / \mathrm{m}^{2}$ and the Poisson's ratio $v=0.451$.

For the Rayleigh damping, two damping coefficients have to be defined: $\alpha_{R}$ and $\beta_{R}$. The relationship between the Rayleigh damping coefficients and the damping ratio is:

$$
\alpha_{R}+\beta_{R} \omega^{2}=2 \omega \xi,
$$

where: $\omega$ is the angular frequency. Solving Eq. (18) for two target frequencies and two target damping ratios, yields:

$$
\begin{gathered}
\alpha_{R}=2 \omega_{1} \omega_{2} \frac{\omega_{1} \xi_{2}-\omega_{2} \xi_{1}}{\omega_{1}^{2}-\omega_{2}^{2}} ; \\
\beta_{R}=2 \frac{\omega_{1} \xi_{1}-\omega_{2} \xi_{2}}{\omega_{1}^{2}-\omega_{2}^{2}} .
\end{gathered}
$$

In this case, for the Rayleigh damping, the target damping ratios are equal $\xi_{1}=\xi_{2}=3 \%$. Unfortunately, there is no other way than selecting two different target frequencies. From the undamped calculations it was 
measured, that most of the pulse energy travels in the frequency range between $f_{1}=5 \mathrm{~Hz}$ and $f_{2}=25 \mathrm{~Hz}$. This leads to the following angular frequencies: $\omega_{1}=2 \pi f_{1}=$ $31.42 \mathrm{rad} / \mathrm{s}$ and $\omega_{2}=2 \pi f_{2}=157.08 \mathrm{rad} / \mathrm{s}$. So the damping coefficients according to Eqs (19) and (20) are respectively $\alpha_{R}=1.571$ and $\beta_{R}=3.183 \cdot 10^{-4}$. Here it should be noted that the results would be different if different target angular frequencies $\omega_{1}$ and $\omega_{2}$ would be selected and this fact just underlines once more the negative points of using the Rayleigh damping for soils.

For the shear damping model, the average shear strain amplitude is in the range of $10^{-5}$. The secant shear modulus $G_{s e c}$ is selected to be equal to the small strain shear modulus $G_{0}$. The shear damping ratio $\xi=3 \%$, results in the following three parameters for the shear damping model: 1) the dimensionless damping coefficient, according to Eq. (2), $X=0.910,2)$ the modified shear modulus, according to Eq. (3), $G_{\text {mod }}=2039 \mathrm{kN} / \mathrm{m}^{2}$ and 3 ) the bulk modulus, defined from the compressional and shear wave velocities, $K=52800 \mathrm{kN} / \mathrm{m}^{2}$.

\section{Results}

Figure 8 demonstrates the horizontal peak displacements and Figure 9 the vertical peak displacements. From these figures, it follows that by applying shear damping, the peak displacements (horizontal and vertical) are higher than by applying Rayleigh damping, especially closer to the source.

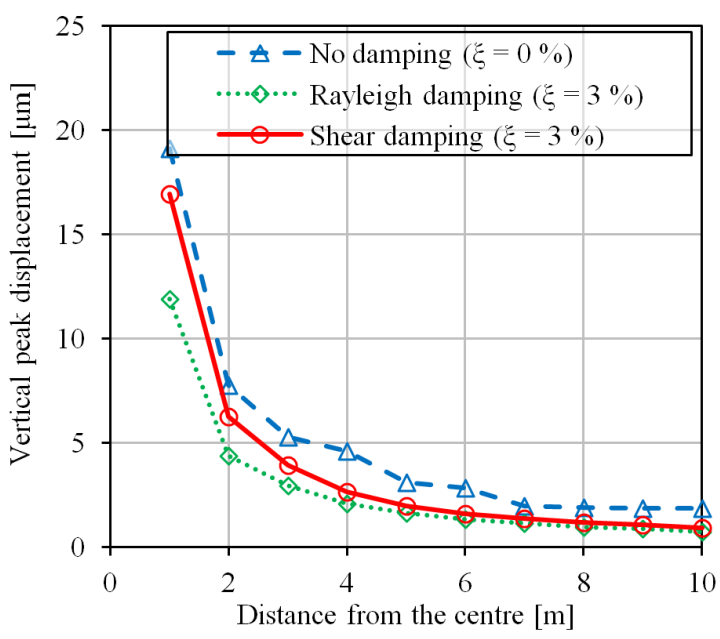

Fig. 8. Peak displacements at different distances in vertical direction

This can be explained by the fact that near the source, there are only compressional and shear waves and the Rayleigh waves are not yet developed. The compressional waves change mostly the volume and hardly the shape. The shear waves change only the shape. The shear damping damps only the changes of the shape, so only the shear waves and a small part of the compressional waves. In contrary, the Rayleigh damping damps all waves equally, so also the displacements of the compressional waves.

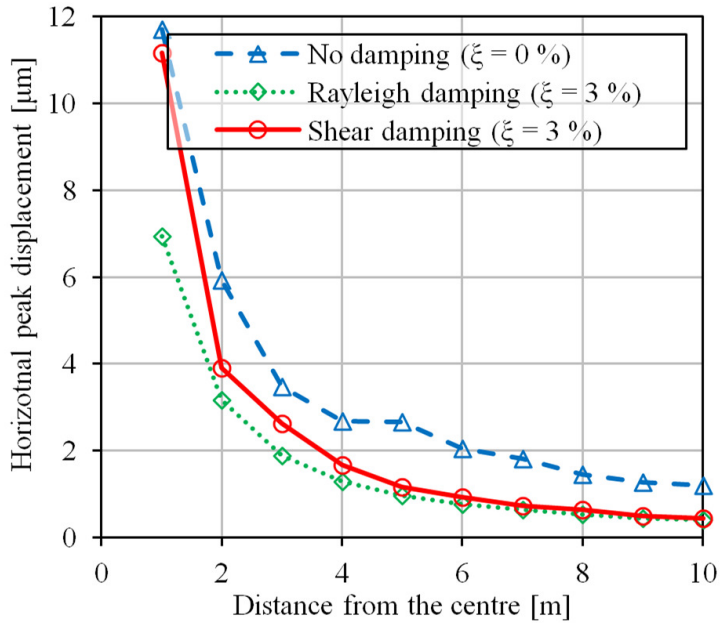

Fig. 9. Peak displacements at different distances in horizontal direction

Further away from the source, the Rayleigh waves are emerging and start to dominate. It causes more changes of the shape rather than the volume; therefore, the differences between the peak displacements, obtained by the two different damping models, are getting smaller. This can be seen from the ratio of the amplitudes calculated by Rayleigh damping to the amplitudes calculated by shear damping at different distances. This is presented in Figure 10.

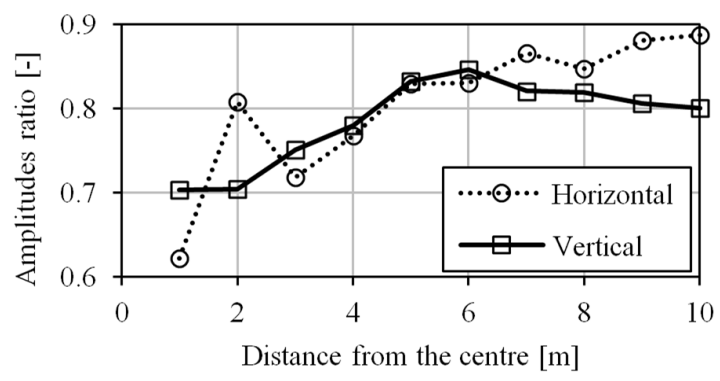

Fig. 10. Ratios between amplitudes calculated by Rayleigh and shear damping models at different distances

In Figure 11, the vertical vibration is shown at two different distances from the centre of the source: at $5 \mathrm{~m}$ and at $10 \mathrm{~m}$. The arrival times of the peak displacements are very close, but not exactly the same.

Comparing the time differences $\Delta t_{R}$ and $\Delta t_{S}$, which correspond to the Rayleigh and shear damping methods respectively, it can be seen, that the signal travels faster (between the same two points) with the shear damping model. This is because the modified shear modulus $G_{\text {mod }}$ is selected for average shear strain amplitudes of $10^{-5}$, whereas the shear strain amplitudes become smaller for larger distances. The shear strain amplitudes decrease due to both the radiation damping and the material damping. This result in a stiffer behaviour of the deviatoric term, therefore the pulse travels faster. Just the opposite occurs closer to the source, where the shear strain deformations are higher than the selected average. 


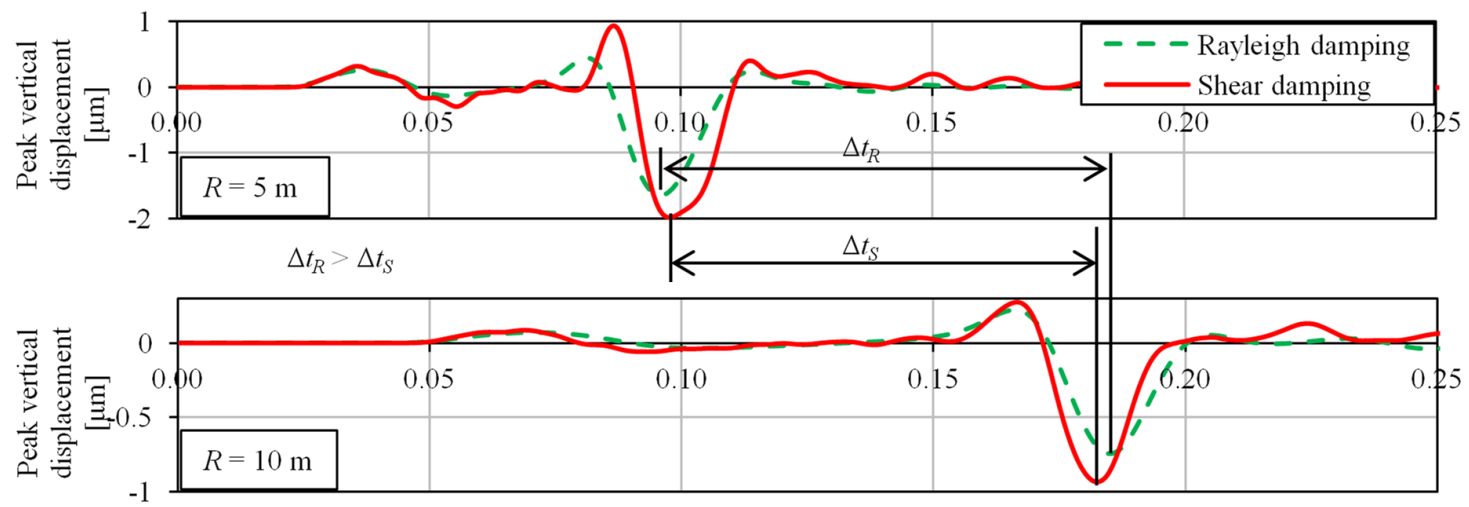

Time $[\mathrm{s}]$

Fig. 11. Record of vertical vibrations at $5 \mathrm{~m}$ and $10 \mathrm{~m}$ distances

The effect of this can be seen by comparing the arrival times at $5 \mathrm{~m}$, where the pulse, calculated by the Rayleigh damping, arrives earlier.

\section{Conclusions}

A 3D shear damping soil model has been developed, which is capable of damping deviatoric strains only and having a constant damping for all frequencies. This shear damping soil model demonstrates a different physical behaviour of the soil than the Rayleigh damping: Closer to the source, the peak displacements are bigger, due to the fact that only the deviatoric part of the strains is damped. This means all basic waves (compressional, shear, Rayleigh) are damped differently. Further away from the source, differences between the peak displacements are smaller than $20 \%$. Also the arrival times obtained by the shear damping model, are shorter in comparison to those of the Rayleigh damping for large distances and just the opposite for small distance. This is due to the selected average shear strain amplitude of the shear damping model. It should be concluded that it is possible to damp small strains physically in a correct manner by using the developed shear damping soil model. However, certain tradeoffs have to be accepted, like variable deviatoric stiffness which results in variable shear and Rayleigh wave speeds.

\section{References}

Abbiss, C. P. 1986. The effects of damping on the interpretation of geophysical measurements, Geotechnique 36(4): 565-580.

http://dx.doi.org/10.1680/geot.1986.36.4.565

Ambrosini, R. D. 2006. Material damping vs. radiation damping in soil-structure interaction analysis, Computers and Geotechnics 33: 86-92. http://dx.doi.org/10.1016/j.compgeo.2006.03.001

Benz, T. 2007. Small-strain stiffness of soils and its numerical consequences: Doctoral thesis. University of Stuttgart.
Bolton, M. D.; Wilson, J. M. R. 1990. Soil stiffness and damping, in Proceedings of The International Conference on Structural Dynamics, Eurodyn '90, University of Bochum, 1: 209-216.

Brinkgreve, R. B. J.; Kappert, M. H.; Bonnier, P. G. 2007. Hysteretic damping in a small-strain stiffness model, in Proceedings of the $10^{\text {th }}$ International Symposium on Numerical Models in Geomechanics (NUMOG X). Rhodes, Greece.

Brinkgreve, R. B. J.; Kumarswamy, S.; Swols, W. M.; Waterman, D.; Chesaru, A.; Bonnier, P. G.; Haxaire, A. 2015. Plaxis material models manual. Delft: Plaxis BV.

Iwasaki, T.; Tatsuoka, F.; Takagi, Y. 1978. Shear moduli of sands under cyclic torsional shear loading, Soils and Foundations 18(1): 39-56.

http://dx.doi.org/10.3208/sandf1972.18.39

Karl, L.; Haegeman, W.; Degrande, G. 2006. Determination of the material damping ratio and the shear wave velocity with the Seismic Cone Penetration Test, Soil Dynamics and Earthquake Engineering 26: 11111126. http://dx.doi.org/10.1016/j.soildyn.2006.03.001

Kramer, S. L. 1996. Geotechnical earthquake engineering. New Jersey: Prentice-Hall.

Okur, D. V.; Ansal, A. 2007. Stiffness degradation of natural fine grained soils during cyclic loading, Soil Dynamics and Earthquake Engineering 27(9): 843-854. http://dx.doi.org/10.1016/j.soildyn.2007.01.005

Richart, F.; Hall, J.; Woods, R. 1970. Vibrations of soils and foundations. Englewood Cliffs (NJ): PrenticeHall.

Sienkiewicz, Z. 1993. Dynamic impedances of a weakly anelastic medium, Earthquake Engineering \& Structural Dynamics 22: 1009-14. http://dx.doi.org/10.1002/eqe.4290221107

Van Baars, S. 2011. Modelling of frictional soil damping in finite element analysis, in International Symposium on Computational Geomechanics, 27-29 April 2011, Cavtat-Dubrovnik, Croatia.

Wolf, J. P. 1985. Dynamic soil-structure-interaction. Englewood Cliffs: Prentice-Hall. 